\title{
Jurnal
}

\section{SNR ANALYSIS ON L-BAND MObile SATELLITE SyStem UNDER BUILDING EFFECT}

\author{
Nur Fadzilah Basria*, Saturi Bacob, W. A. Wan Zainal Abidinc and \\ Saufi Affendy Basric
}

aPreparatory Centre for Science and Technology, Universiti Malaysia Sabah, 88400 Kota Kinabalu, Sabah, Malaysia bPhysics with Electronics Programme, Faculty of Science and Natural Resources, Universiti Malaysia Sabah, 88400 Kota Kinabalu, Sabah, Malaysia

cDepartment of Electronics Engineering, Faculty of Engineering, Universiti Malaysia Sarawak, 93400 Kota Samarahan, Sarawak, Malaysia
Article history

Received

14 January 2015

Received in revised form

31 Mac 2015

Accepted

09 June 2015

*Corresponding author dilabasri@ums.edu.my

\section{Graphical abstract}

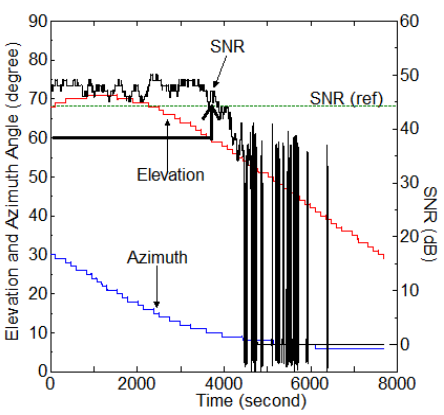

\begin{abstract}
This paper presents the method to analyse the effect of building on the L-band Mobile Satellite (MS) system using low-power Global Positioning System (GPS) receiver. The method includes measurement, experimental and data analysis. The analysis of the signal performance under building effect measurement was carried out with respect to the signal to noise ratio (SNR), elevation and azimuth angle. In measurement method, the National Marine Electronics Association (NMEA) sentences obtained from the satellite via GPS receiver was used to get the signal propagation parameters. The NMEA Extractor used to extract the NMEA data using $\mathrm{C}++$ programming language and Ngraph software to construct the graphical presentation for analysis method. The analysis shows relationship between SNR and elevation and azimuth angle. The comparison between open space and building effect was carried out and the results have shown that the presence of the building affect the quality of the satellite signal received.
\end{abstract}

Keywords: MS system, GPS, building effect, L-band, SNR, elevation angle, azimuth angle

\subsection{INTRODUCTION}

MS system refers to transmission via satellite from nonstationary's transmitter and receivers. MS systems are suitable for mobile user and more flexible in terms of navigation systems on moving vehicles [1]. The main advantage of MS system compare to Fixed Satellite (FS) system is how the coverage signal of MS system is spotty over rural area and it is available for movement transceiver. Therefore, the MS system is suitable to system of mobile user and more flexible in terms of navigation system on moving vehicles.

GPS is the system in navigation in an accuracies range from a few millimetres until 15 meters [2]. Constellation of GPS is at least 24 Medium Earth Orbit
(MEO) satellite that transmit microwave signal in six orbital planes and requiring a minimum of four satellites per planes. GPS satellites are orbiting the earth about 12,000 miles above the earth with speed of 7,000 miles an hour [3]. It makes two complete orbits in 24 hour. The GPS system enables the user to locate the accurate position by consulting radio receiver.

The main factors that cause the propagation impairment in MS system are shadowing effect, ionospheric effect and multipath fading. Shadowing effect such as building, tree and terrain is interacted with wave propagation via diffraction, reflection and scattering. The signal that was blocked by building caused the signal propagated into different path before it arrived at receiver. These multipath signals 